\title{
Structural basis of ribosomal frameshifting during translation of the SARS-CoV-2 RNA genome
}

\author{
Pramod R. Bhatt, ${ }^{1,2,3}$, Alain Scaiola ${ }^{1} \uparrow$, Gary Loughran $^{2}$, Marc Leibundgut $^{1}$, Annika Kratzel ${ }^{4,5,6}$, Romane $^{1}$ Meurs $^{7}$, \\ René Dreos ${ }^{7}$, Kate M. O'Connor ${ }^{2}$, Angus McMillan ${ }^{8}$, Jeffrey W. Bode ${ }^{8}$, Volker Thiel ${ }^{4,5}$, David Gatfield ${ }^{7}$, \\ John F. Atkins ${ }^{2,3,9 *}$, Nenad Ban ${ }^{1 *}$ \\ ${ }^{1}$ Department of Biology, Institute of Molecular Biology and Biophysics, ETH Zurich, Zurich, Switzerland. 2School of Biochemistry and Cell Biology, University College Cork, \\ Cork T12 XF62, Ireland. ${ }^{3}$ School of Microbiology, University College Cork, Cork T12 K8AF, Ireland. ${ }^{4}$ Institute of Virology and Immunology, University of Bern, Bern, \\ Switzerland. ${ }^{5}$ Department of Infectious Diseases and Pathobiology, Vetsuisse Faculty, University of Bern, Bern, Switzerland. ${ }^{6}$ Graduate School for Cellular and Biomedical \\ Sciences, University of Bern, Bern, Switzerland. ${ }^{7}$ Center for Integrative Genomics, Génopode, University of Lausanne, 1015 Lausanne, Switzerland. ${ }^{8}$ Laboratorium für \\ Organische Chemie, Department of Chemistry and Applied Biosciences, ETH Zurich, Zurich, Switzerland. ${ }^{9} \mathrm{MRC}$ Laboratory of Molecular Biology, Cambridge CB2 0QH, UK. \\ †These authors contributed equally to this work. *Corresponding author. Email: j.atkins@ucc.ie (J.F.A.); ban@mol.biol.ethz.ch (N.B.)
}

Programmed ribosomal frameshifting is a key event during translation of the SARS-CoV-2 RNA genome allowing synthesis of the viral RNA-dependent RNA polymerase and downstream proteins. Here we present the cryo-electron microscopy structure of a translating mammalian ribosome primed for frameshifting on the viral RNA. The viral RNA adopts a pseudoknot structure that lodges at the entry to the ribosomal mRNA channel to generate tension in the mRNA and promote frameshifting, whereas the nascent viral polyprotein forms distinct interactions with the ribosomal tunnel. Biochemical experiments validate the structural observations and reveal mechanistic and regulatory features that influence frameshifting efficiency. Finally, we compare compounds previously shown to reduce frameshifting with respect to their ability to inhibit SARS-CoV-2 replication, establishing coronavirus frameshifting as a target for antiviral intervention.

Ribosomal frameshifting, a process during which the reading frame of translation is changed at the junction between open reading frames $1 \mathrm{a}$ and $1 \mathrm{~b}$, is one of the key events during translation of the severe acute respiratory syndrome coronavirus 2 (SARS-CoV-2) positive sense single-stranded RNA genome. This programmed -1 translational frameshifting is conserved in all coronaviruses and is necessary for synthesis of viral RNA-dependent RNA polymerase (RdRp or Nsp12) and downstream viral non-structural proteins encoding core enzymatic functions involved in capping of viral RNA, RNA modification and processing, and RNA proof-reading (1). Although the translational machinery typically prevents frameshifting as a potential source of one of the most disruptive errors in translation $(2,3)$, many viruses rely on programmed ribosomal frameshifting to expand and fine-tune the repertoire and stoichiometry of expressed proteins (4).

Programmed - 1 frameshifting in SARS-related coronaviruses occurs at the slippery sequence U_UUA_AAC in the context of a 3 ' stimulatory RNA sequence that was predicted to form a 3-stemmed pseudoknot structure (5), and in parallel was independently tested by our lab and others $(6-8)$. The frameshifting occurs with high efficiency (25-75\%) depending on the system used $(6,7,9-11)$ and changes the reading frame to UUU_AAA_C (12) (Fig. 1A). Consequently, two viral polyproteins are synthesized, one encoded by the ORFla when frameshifting does not take place, whereas ORFlab is expressed as a result of frameshifting. Translation of ORFla produces polyprotein 1a ending with Nsp10 followed by the short Nsp11. On the other hand, when the frameshift occurs, the polyprotein lab is generated, which contains almost 2700 additional amino acids and in which the viral RdRp, Nsp12, is produced after Nsp10 as a consequence of translation in the -1 frame. A putative secondary structure element in the viral RNA that forms a loop upstream of the shift site has been proposed to play an attenuating role in frameshifting and is referred to as the $5^{\prime}$ attenuator loop (8). Maintaining the precise level of coronavirus frameshifting efficiency is crucial for viral infectivity, evidenced by the remarkable fact that mutation of a single nucleotide in the frameshifting region of the SARS-CoV-1 RNA results in a concomitant abrogation of viral replication (13). Therefore, the importance of 3 -stemmed pseudoknot-dependent -1 ribosomal frameshifting for the propagation of SARS-related coronaviruses, a process that has not been seen to occur on any endogenous human transcript in human cells, presents itself as an opportune drug-target with minimal tolerance for drug-resistant mutations.

Due to its importance in the life cycle of many important viruses and coronaviruses in particular, programmed frameshifting has been extensively studied using a range of structural and functional approaches (4). The structure of a $3^{\prime}$ stimulatory pseudoknot in isolation or in context of the viral genome has been proposed recently by various groups using techniques that include molecular dynamics, nuclease 
mapping, in vivo selective 2 '-hydroxyl acylation analyzed by primer extension (SHAPE), nuclear magnetic resonance (NMR) and cryo-electron microscopy (cryo-EM) (7, 14-17). Furthermore, a ribosomal complex with a frameshift stimulatory pseudoknot from the avian infectious bronchitis virus was reported at low resolution (18). Here, to provide a structural and mechanistic description of the events during ribosomal frameshifting, we investigated mammalian ribosomes captured in distinct functional states during translation of a region of SARS-CoV-2 genomic RNA where -1 programmed frameshifting occurs.

\section{Structure determination of a frameshifting-primed ribosomal complex}

We captured a 0 frame, pre-frameshift ribosomal complex by introducing a stop codon in place of the second codon of the slippery site (U_UUA_AAC to U_UUA_UAA) (Fig. 1A) and adding mutant eukaryotic Release Factor 1 [eRF1 (AAQ)] that is unable to release the nascent polypeptide. Translating complexes were prepared in an in vitro translation reaction using an in-house generated rabbit reticulocyte lysate (RRL) system that supported efficient frameshifting in the previously reported range of around 50\% (19) according to dual luciferase experiments (see methods). The ribosomes were programmed with mRNA encoding an affinity tag and harboring a region of the SARS-CoV-2 genome that encodes proteins Nsp10 (C terminus), Nsp11 and the majority of Nsp12. Western blotting showed that when using the WT RNA template, frameshifting was efficient, while the stop codon mutation prevented frameshifting and led to ribosome pausing. This effect was further enhanced when eRF1 (AAQ) was present in excess over endogenous wild type eRF1 (Fig. 1B).

The cryo-EM 3D reconstruction of ribosome-nascent chain complexes (RNCs) affinity purified from the reactions supplemented with eRF1 (AAQ) revealed two distinct ribosomal complexes captured in the process of translating the slippery sequence (figs. S1 and S2). One represented a termination complex that contained the ATP-binding cassette transporter 1 (ABCE1) known to be involved in termination and recycling together with mutant eRF1 interacting with the stop codon (fig. S3). The second reconstruction resolved translating $80 \mathrm{~S}$ ribosomes containing $\mathrm{P}$ - and E-site tRNAs bound (fig. S2). This reconstruction at $2.2 \AA$ resolution allowed us to build the most accurate structure of a mammalian $80 \mathrm{~S}$ ribosome so far and directly visualize many protein and virtually all rRNA modifications identified for the human ribosome based on quantitative mass spectrometry and as interpreted in a recent human ribosome structure $(20,21)$, consistent with the complete conservation of all modified residues between rabbit and human rRNAs (figs. S4 and S5; and tables S1 to S3). Importantly, this reconstruction also featured additional density at the entrance to the mRNA channel suggestive of a structured RNA, which after focused classification revealed a prominent density for a complete $3^{\prime}$ frameshifting stimulatory pseudoknot at the entry of the mRNA channel on the 40S subunit (Fig. 1, C and D). The resolution of this reconstruction ranged from $2.4 \AA$ at the core of the ribosome to $\sim 7 \AA$ at the periphery, where the most flexible regions of the pseudoknot are located (figs. S2 and S6). Based on the high-resolution maps that allowed visualization of the codon-anticodon interactions and modifications in the tRNA (Fig. 1E and fig. S6, A and B), we could unequivocally determine that a Phe-tRNA(Phe) was bound at the P-site (22). The mRNA does not adopt any unusual structure in the Asite of the ribosome as was observed for the HIV-1 frameshifting sequence visualized on the bacterial ribosome (23). This implied that the ribosome is paused by the downstream pseudoknot located at the entrance to the mRNA channel such that the P-site tRNA interacts with the UUU codon just prior to the first codon, UUA, of the slippery site (Fig. 2A).

\section{The pseudoknot causes ribosomal pausing prior to -1 frameshifting}

The observation that the pseudoknot acts as an obstacle to slow down translation as the ribosome approaches the slippery site is mechanistically reasonable. Since the pseudoknot is a stable structural element in the mRNA, it will resist unfolding and consequently generate a back-pull on the viral RNA, resulting in an increased chance of -1 frameshifting as the tRNAs are translocated. A pause in translocation at a codon that precedes the slippery site, characterized by a $>10$ times longer occupancy prior to the slippage event, was observed in an analogous case of heptanucleotide - 1 frameshifting on the bacterial dnaX gene using single molecule experiments (24). According to this model, it would be anticipated that a further round of translocation results in unwinding of Stem 1 of the downstream stimulatory pseudoknot structure. Consistently, in our structure of the eRF1 (AAQ)bound ribosome that advanced one codon further along the mRNA, no clear secondary structure is visible at the entrance to the mRNA channel as the mRNA now becomes disordered at this position (figs. S1 and S3, A and B).

In order to investigate the slowdown of translation on the wild type slippery sequence, we performed disome footprint profiling, a method to identify translational pause sites through the analysis of transitory ribosome collisions (25-27) (see methods). Notably, recent studies using conventional ribosome profiling methodology reported a lack in monosome footprint coverage across the frameshifting region on the SARS-CoV-2 RNA $(11,28)$ - possibly because ribosomes in this area became trapped in temporary collisions. Moreover, the highly structured pseudoknot at the entry to the mRNA channel would likely preclude efficient trimming by RNase I, the enzyme used for footprint generation, further reducing 
efficient monosome footprint capture. Using a modified nuclease treatment protocol (see methods) that recovered monosome footprints from the frameshift region (Fig. 3, A and C), our experiments revealed that ribosome collisions occur as a result of ribosomal pausing at the same position that is observed in the structure of the pseudoknot-engaged ribosome (Fig. 3, B and D). Apparently, although the base substitutions creating a stop codon in the $3^{\prime}$ adjacent slippery site did not change the features of pausing, it increased the dwell time of the ribosomes at the pause site sufficiently to allow visualization in the cryo-EM experiment.

The results of our disome profiling experiments prompted us to structurally investigate disomes by cryo-EM. We were able to visualize the pseudoknot-paused ribosome followed by a closely trailing ribosome. Upon focused refinement, we obtained a high-resolution (3.1 $\AA$ ) structure of the trailing ribosome in a rotated state (fig. S1). In congruence with our estimated positioning of the ribosomes in disome profiling (Fig. 3D), the purine-pyrimidine pattern of codon-anticodon pairs in the structure of the colliding ribosome revealed that the pause occurs with CCC and AUG triplets in the P- and Asites, respectively (Fig. 3C).

\section{The SARS-CoV-2 RNA pseudoknot specifically interacts with ribosomal proteins and $18 S$ rRNA}

The intermediate local resolution (5-7 $\AA$ ) of the cryo-EM map in the area of the pseudoknot allowed us to visualize the overall fold of the RNA and readjust its previously predicted secondary structure $(14-17,19)$ (Fig. 1, C, D, and F). The stimulatory pseudoknot forms an H-type pseudoknot with Stem 1 and Stem 2 coaxially stacked on top of each other to form a quasi-continuous helix, while Stem 3 stands out almost perpendicular to this plane (Figs. 1D and 2B). This corkscrew-like formation provides a bulky and well-structured obstacle wedged at the mRNA entry channel, having the potential to resist unwinding by the helicase activity of the ribosome and generating tension on the upstream mRNA up to the decoding center. Stem 1 of the pseudoknot forms a 9 bp helix which is GC rich at the bottom (Fig. 1F). The penultimate nucleotides of the 'spacer region' prior to Stem 1 are located at the mRNA entry tunnel, where they interact with several basic residues in the C-terminal domain of uS3 on one side and are supported by uS5 from the other, with an additional weak contact contributed by the C-terminal end of eS30. uS3 and eS30 are primary components of the ribosome helicase and uS5 has been proposed to be a component of the ribosomal helicase processivity clamp at the mRNA entry site $(29,30)$. The observed distance between the P-site UUU codon and Stem 1 of the pseudoknot underscores the critical dependence of the frameshifting efficiency on the length of the spacer region (31). Translocation to the next codon would place the frameshifting codon UUA into the P-site, with a simultaneous increase in the tension of the mRNA and unwinding of the GC-rich base of Stem 1 upon entering into the mRNA entry channel, comparable to the situation when the ribosome proceeds to the engineered stop codon as observed in our eRF1 (AAQ)-stalled structure (fig. S3).

The pseudoknot structure also reveals a hitherto unobserved and possibly unappreciated role for the distal site of the mRNA entrance channel in helicase activity. While mRNA unwinding studies outside the mRNA entrance channel have so far implicated only a helix in the C-terminal domain of uS3 (32), we notice that Loop 1 of the pseudoknot contacts the N-terminal domain of uS3 as well as the C-terminal tail of eS10 (Fig. 2B and fig. S6D), whereas the flippedout base G13486 in this loop forms specific interactions (Fig. 2B). Furthermore, as the pseudoknot is located at the entry to the mRNA channel, helix h16 of the $18 \mathrm{~S}$ rRNA is noticeably pushed outwards due to a direct contact with the minor groove of Stem 1 (Fig. 2B and fig. S7A). Since the pseudoknot wedges between the head and the body of the small ribosomal subunit, it would restrict their relative motions that need to take place during translocation. This is consistent with the studies on dynamics of coronavirus frameshifting, which revealed that the mechanism of -1 frameshifting involves restriction of small subunit head motion (33).

The structure also reveals another key aspect of the architecture of the pseudoknot as the ribosome encounters it. The start of the pseudoknot is shifted relative to the predicted secondary structure $(14-17,19)$ by two nucleotides. The two opposed nucleotides, which were assumed to base pair with Stem 1, are actually forming the start of Stem 3 by pairing with bases predicted to be in the single-stranded linker 2 (Fig. $1 \mathrm{~F}$ and fig. S7, B and C). Our cryo-EM density reveals that Loop 3 accommodates a total of 4 nucleotides, three of which were originally attributed to Stem 2 . Thus, we observe that Loop 3 is shifted and expanded relative to the initially predicted secondary structures $(14-17,19)$.

To functionally support our structural findings and confirm the nature and specificity of the pseudoknot interactions, we performed structure-guided mutagenesis experiments using dual luciferase reporter assays in HEK293T cells (see methods) and monitored the frameshifting efficiency relative to the WT (Fig. 2C). Mutation of G13486 of Loop 1 to another purine reduced the frameshifting efficiency to $30 \%$ of the WT level, and mutation of this base to a pyrimidine further reduced frameshifting to 15\%. As expected from our structural data, deletions of the nucleotides of the spacer regions also had a deteriorating effect on frameshifting. Loss of Loop 1 entirely abolished frameshifting. Deletion of a single nucleotide of Loop 3 in agreement with its proposed role in forming the base pairing interactions diminished the frameshifting rate to $25 \%$ of the WT level. Loss of the entire Loop 3 reduced frameshifting to $10 \%$ of WT levels. 


\section{Frameshifting efficiency depends on the position of the " 0 " frame stop codon}

In SARS-CoV-2, the 0 frame stop codon is located 5 codons downstream of the frameshift site and is a constituent of Stem 1 . The placement of the stop codon in such proximity to the frameshift site is a common feature in coronaviruses, and its presence in a critical region of the stimulatory pseudoknot prompted us to probe the effect of the distance of the 0 frame stop codon on frameshifting. To this end, knowledge of the 3D structure of the pseudoknot helped us to confidently manipulate the stop codon without hampering pseudoknot formation. We introduced mutations to incrementally extend the stop codon from the WT position and to completely remove the occurrence of a stop codon in the 0 frame (Fig. 2D and fig. S8). While introducing a stop codon 6 nucleotides downstream of the WT position only marginally decreased the frameshifting rate ( $98 \%$ of WT), a stronger attenuation was observed when the distance of the stop codon was increased to 15 nucleotides from the WT stop ( $80 \%$ of WT). Finally, removal of the stop codon by two different point mutations led to a reduction of frameshifting efficiency to $50 \%$ of WT levels. To test whether reduced ribosomal loading rescues the effect of stop codon removal, we analyzed the frameshifting efficiency in the context of weaker initiation codons such as CUG and AUU (Fig. 2D). These constructs led to a $45 \%$ rescue of the reduction in frameshifting compared to stop codon mutants initiating at an AUG start.

Taken together, these observations suggest that the stop codon position plays an important role in maintaining optimum frameshift efficiency. We propose that the stop codon serves to prevent the closely trailing ribosome from encountering a viral RNA that was unfolded by the leading ribosome. In this case, upon encountering a stop codon, termination and subunit disassembly will occur, which will provide an opportunity for the pseudoknot to refold without the constraints of the mRNA channel (see Conclusions). According to this model, although the wild type stop codon will make the frameshifting efficiency less sensitive to ribosome loading in the "no-frameshifting" scenario, the frameshifting events that occur following a -1 frameshift will nevertheless be more likely when the ribosomes are spaced further apart. Our measurements of the efficiency of frameshifting for the wild type sequence in the context of different rates of translation initiation are in agreement with this hypothesis (fig. S9). This mechanism, consistent with our biochemical data, increases the efficiency of frameshifting to the levels required by SARSCoV-2 and may be used by viruses in general when high-efficiency frameshifting is required.

\section{Nascent chain forms specific interactions with the ribosomal exit tunnel}

Strikingly, in the reconstruction of the paused translating ribosome, the nascent chain that corresponds to the viral polyprotein was visible along the entire length of the ribosomal exit tunnel (Fig. 4A). The density corresponded to the C-terminal region of Nsp10, which is the activator of the viral proofreading exonuclease and N7-methyltransferase Nsp14 $(34,35)$, and then (depending on the frameshifting event) continues as either the viral RNA-dependent RNA polymerase Nsp12 (6) or as protein Nsp11, whose function is yet unknown (Figs. 1A and 4B). The nascent chain makes several specific interactions with the ribosomal tunnel, one of which is at the constriction site where arginine 4387 of Nsp10 interacts with A1555 of the 28S rRNA [corresponding to A1600 in humans, numbering according to PDB 6EK0 (36)] and is stabilized by the preceding leucine 4386 (Fig. 4C). Notably, these two amino acids are highly conserved across multiple coronaviruses (Fig. 4G), although they are located in the unstructured C-terminal region of Nsp10 and therefore considered not to be important for the fold of the protein (37).

Further down the tunnel, the C-terminal end of Nsp10 adopts a partially folded zinc finger motif (Fig. 4, D and E), which upon superposition reveals similarity with the corresponding fully folded C-terminal domain previously observed in the crystal structure of SARS-CoV-1 Nsp10 (37). Tryptophan 4376 located between the two pairs of cysteines that form the zinc finger stacks with A2261 (A2418), an interaction that might serve to promote the change of nascent chain direction and facilitate folding of the zinc finger at the end of the exit tunnel. Co-translational events, such as insertion of a transmembrane domain at the exit of the ribosomal tunnel, was shown to promote -1 ribosomal frameshifting in alphaviruses (38).

To investigate whether the observed contacts between the nascent chain and the ribosomal tunnel are specific, and whether these interactions and co-translational folding of Nsp10 might play a role in modulating the frameshifting process, we employed our dual luciferase reporter assay to measure the frameshifting efficiency of WT and mutant nascent chain sequence constructs. As our measurements in HEK293T cells did not reveal an appreciable change of frameshift efficiency, we carried out the same experiments in vitro using RRL to monitor the effects in a single mRNA setup. Replacement of the entire nascent chain with an unrelated sequence leads to a $35 \%$ increase in frameshifting (Fig. $4 \mathrm{~F})$. Importantly, this effect was provoked by the change in peptide sequence and not simply by the loss of the $5^{\prime}$ attenuator loop, given that a reporter containing silent attenuator loop mutations resulted in only a slight increase in frameshifting (Fig. 4F). Mutation of the leucine 4386 and arginine 4387 to alanine led to a considerable (30\%) increase in frameshifting (Fig. 4, F and G), implying that these nascent chain interactions with the ribosomal exit tunnel play an important role in regulating frameshifting levels, possibly 
mechanistically akin to the well-studied SecM stalling system in bacteria (39), where it was shown that co-translational folding and the translocon-induced mechanical force can rescue the stall induced by interactions between the nascent chain and the ribosomal tunnel (40). These observations also suggest that any cellular nascent-chain factors $(41,42)$ might influence the rate of frameshifting.

\section{Inhibition of viral replication by a compound that targets the SARS-CoV-2 pseudoknot}

The sensitivity of the coronavirus to the finely controlled frameshifting levels (13) may present an opportunity to develop compounds that interfere with the frameshifting process and thus inhibit replication of the virus. Using computational modeling and reporter assays, compounds that have been predicted to bind the pseudoknot and inhibit SARS-CoV-2 frameshifting were described $(19,43)$, but never tested with respect to their ability to inhibit viral replication. Furthermore, the fluoroquinolone compound merafloxacin was recently reported to also inhibit -1 frameshifting efficiency of SARS-CoV-2 and other betacoronaviruses (44). To demonstrate that the inhibition of frameshifting is a plausible strategy for drug development, we compared two of the previously described compounds with respect to their ability to reduce viral levels in infected African green monkey VeroE6 cells (fig. S10 and methods). Our experiments show that merafloxacin is a better candidate compound as it showed a concentration dependent inhibition of frameshifting, whereas, contrary to earlier reports $(19,43)$ MTDB under our experimental conditions did not specifically inhibit frameshifting (fig. S10). The two compounds showed no cellular toxicity and resulted in a 3 to 4 orders of magnitude reduction of SARS-CoV-2 titer, with the half maximal inhibitory concentration (IC50) of $48 \mu \mathrm{M}$ for MTDB and an order of magnitude higher efficacy of merafloxacin with an IC50 of $4.3 \mu \mathrm{M}$ (fig. S10). Since MTDB did not appear to affect frameshifting in our reporter construct experiments in vitro and in vivo, it is possible that it inhibits SARS-CoV-2 replication by a different mechanism. Although the potency range for these compounds is not what would be expected from potential drug candidates, it nevertheless provides a starting point for high-throughput screening and establishes that frameshifting is a viable target for therapeutic intervention against SARS-CoV-2.

\section{Conclusions}

Our results provide a mechanistic description of frameshifting that occurs during translation of the SARS-CoV-2 genome and reveal the features that may be exploited by the virus to finely control the stoichiometry of viral proteins at different stages of infection (Fig. 5). Interfering with the frameshifting process at the level of nascent chain interactions with the ribosomal tunnel, at the level of RNA folding that leads to the formation of the frameshift stimulatory pseudoknot, or to perturb the interactions between the pseudoknot and the mRNA channel, represent a viable strategy in our search for new drugs against SARS-CoV-2, the virus that is currently causing the global COVID-19 pandemic. Our results will also be useful for understanding the mechanism of programmed ribosomal "-1" frameshifting (4) including that employed by many other medically important viruses.

\section{REFERENCES AND NOTES}

1. P. V'kovski, A. Kratzel, S. Steiner, H. Stalder, V. Thiel, Coronavirus biology and replication: Implications for SARS-CoV-2. Nat. Rev. Microbiol. 19, 155-170 (2021). doi:10.1038/s41579-020-00468-6 Medline

2. J. Parker, Errors and alternatives in reading the universal genetic code. Microbiol. Rev. 53, 273-298 (1989). doi:10.1128/MR.53.3.273-298.1989 Medline

3. J. M. Ogle, A. P. Carter, V. Ramakrishnan, Insights into the decoding mechanism from recent ribosome structures. Trends Biochem. Sci. 28, 259-266 (2003). doi:10.1016/S0968-0004(03)00066-5 Medline

4. J. F. Atkins, G. Loughran, P. R. Bhatt, A. E. Firth, P. V. Baranov, Ribosomal frameshifting and transcriptional slippage: From genetic steganography and cryptography to adventitious use. Nucleic Acids Res. 44, 7007-7078 (2016). doi:10.1093/nar/gkw530 Medline

5. F. Dos Ramos, M. Carrasco, T. Doyle, I. Brierley, Programmed -1 ribosomal frameshifting in the SARS coronavirus. Biochem. Soc. Trans. 32, 1081-1083 (2004). doi:10.1042/BST0321081 Medline

6. P. V. Baranov, C. M. Henderson, C. B. Anderson, R. F. Gesteland, J. F. Atkins, M. T. Howard, Programmed ribosomal frameshifting in decoding the SARS-CoV genome. Virology 332, 498-510 (2005). doi:10.1016/j.virol.2004.11.038 Medline

7. E. P. Plant, G. C. Pérez-Alvarado, J. L. Jacobs, B. Mukhopadhyay, M. Hennig, J. D. Dinman, A three-stemmed mRNA pseudoknot in the SARS coronavirus frameshift signal. PLOS Biol. 3, e172 (2005). doi:10.1371/journal.pbio.0030172 Medline

8. M. C. Su, C. T. Chang, C. H. Chu, C. H. Tsai, K. Y. Chang, An atypical RNA pseudoknot stimulator and an upstream attenuation signal for -1 ribosomal frameshifting of SARS coronavirus. Nucleic Acids Res. 33, 4265-4275 (2005). doi:10.1093/nar/gki731 Medline

9. I. Brierley, P. Digard, S. C. Inglis, Characterization of an efficient coronavirus ribosomal frameshifting signal: Requirement for an RNA pseudoknot. Cell 57. 537-547 (1989). doi:10.1016/0092-8674(89)90124-4 Medline

10. N. Irigoyen, A. E. Firth, J. D. Jones, B. Y. W. Chung, S. G. Siddell, I. Brierley, Highresolution analysis of coronavirus gene expression by RNA sequencing and ribosome profiling. PLOS Pathog. 12, el005473 (2016). doi:10.1371/journal.ppat.1005473 Medline

11. Y. Finkel, O. Mizrahi, A. Nachshon, S. Weingarten-Gabbay, D. Morgenstern, Y. Yahalom-Ronen, H. Tamir, H. Achdout, D. Stein, O. Israeli, A. Beth-Din, S. Melamed, S. Weiss, T. Israely, N. Paran, M. Schwartz, N. Stern-Ginossar, The coding capacity of SARS-CoV-2. Nature 589, 125-130 (2021). doi:10.1038/s41586-020-2739-1 Medline

12. V. Thiel, K. A. Ivanov, Á. Putics, T. Hertzig, B. Schelle, S. Bayer, B. Weißbrich, E. J. Snijder, H. Rabenau, H. W. Doerr, A. E. Gorbalenya, J. Ziebuhr, Mechanisms and enzymes involved in SARS coronavirus genome expression. J. Gen. Virol. 84, 2305-2315 (2003). doi:10.1099/vir.0.19424-0 Medline

13. E. P. Plant, R. Rakauskaite, D. R. Taylor, J. D. Dinman, Achieving a golden mean: Mechanisms by which coronaviruses ensure synthesis of the correct stoichiometric ratios of viral proteins. J. Virol. 84, 4330-4340 (2010). doi:10.1128/JVI.02480-09 Medline

14. R. Rangan, I. N. Zheludev, R. J. Hagey, E. A. Pham, H. K. Wayment-Steele, J. S. Glenn, R. Das, RNA genome conservation and secondary structure in SARS-CoV2 and SARS-related viruses: A first look. RNA 26, 937-959 (2020). doi:10.1261/rna.076141.120 Medline

15. K. Zhang, I. N. Zheludev, R. J. Hagey, M. T. Wu, R. Haslecker, Y. J. Hou, R. Kretsch, G. D. Pintilie, R. Rangan, W. Kladwang, S. Li, E. A. Pham, C. Bernardin-Souibgui, R. S. Baric, T. P. Sheahan, V. D Souza, J. S. Glenn, W. Chiu, R. Das, Cryo-electron 
microscopy and exploratory antisense targeting of the 28-kDa frameshift stimulation element from the SARS-CoV-2 RNA genome. bioRxiv 2020.07.18.209270 [Preprint]. 20 July 2020; https://doi.org/10.1101/2020.07.18.209270.

16. T. C. T. Lan, M. F. Allan, L. E. Malsick, S. Khandwala, S. Y. Nyeo, Y. Sun, J. U. Guo, M. Bathe, A. Griffiths, S. Rouskin, Insights into the secondary structural ensembles of the full SARS-CoV-2 RNA genome in infected cells. bioRxiv 2020.06.29.178343 [Preprint]. 19 February 2021; https://doi.org/10.1101/2020.06.29.178343.

17. N. C. Huston, H. Wan, M. S. Strine, R. de Cesaris Araujo Tavares, C. B. Wilen, A. M. Pyle, Comprehensive in vivo secondary structure of the SARS-CoV-2 genome reveals novel regulatory motifs and mechanisms. Mol. Cell 81, 584-598.e5 (2021). doi:10.1016/i.molcel.2020.12.041 Medline

18. O. Namy, S. J. Moran, D. I. Stuart, R. J. C. Gilbert, I. Brierley, A mechanical explanation of RNA pseudoknot function in programmed ribosomal frameshifting. Nature 441, 244-247 (2006). doi:10.1038/nature04735 Medline

19. J. A. Kelly, A. N. Olson, K. Neupane, S. Munshi, J. San Emeterio, L. Pollack, M. T. Woodside, J. D. Dinman, Structural and functional conservation of the programmed -1 ribosomal frameshift signal of SARS coronavirus 2 (SARS-CoV-2). J. Biol. Chem. 295, 10741-10748 (2020). doi:10.1074/ibc.AC120.013449 Medline

20. M. Taoka, Y. Nobe, Y. Yamaki, K. Sato, H. Ishikawa, K. Izumikawa, Y. Yamauchi, K. Hirota, H. Nakayama, N. Takahashi, T. Isobe, Landscape of the complete RNA chemical modifications in the human 80 S ribosome. Nucleic Acids Res. 46, 92899298 (2018). doi:10.1093/nar/gky811 Medline

21. W. Li, S. T. L. Chang, F. R. Ward, J. H. D. Cate, Selective inhibition of human translation termination by a drug-like compound. Nat. Commun. 11, 4941 (2020). doi:10.1038/s41467-020-18765-2 Medline

22. G. Keith, G. Dirheimer, The primary structure of rabbit, calf and bovine liver tRNAPhe. Biochim. Biophys. Acta 517, 133-149 (1978). doi:10.1016/00052787(78)90041-2 Medline

23. C. Bao, S. Loerch, C. Ling, A. A. Korostelev, N. Grigorieff, D. N. Ermolenko, mRNA stem-loops can pause the ribosome by hindering A-site tRNA binding. eLife $\mathbf{9}$, e55799 (2020). doi:10.7554/eLife.55799 Medline

24. J. Choi, S. O'Loughlin, J. F. Atkins, J. D. Puglisi, The energy landscape of -1 ribosomal frameshifting. Sci. Adv. 6, eaax6969 (2020). doi:10.1126/sciadv.aax6969 Medline

25. P. Han, Y. Shichino, T. Schneider-Poetsch, M. Mito, S. Hashimoto, T. Udagawa, K. Kohno, M. Yoshida, Y. Mishima, T. Inada, S. Iwasaki, Genome-wide survey of ribosome collision. Cell Rep. 31, $107610 \quad$ (2020). doi:10.1016/i.celrep.2020.107610 Medline

26. A. B. Arpat, A. Liechti, M. De Matos, R. Dreos, P. Janich, D. Gatfield, Transcriptomewide sites of collided ribosomes reveal principles of translational pausing. Genome Res. 30, 985-999 (2020). doi:10.1101/gr.257741.119 Medline

27. S. Meydan, N. R. Guydosh, Disome and trisome profiling reveal genome-wide targets of ribosome quality control. Mol. Cell 79, 588-602.e6 (2020). doi:10.1016/i.molcel.2020.06.010 Medline

28. M. Puray-Chavez, K. Tenneti, H. R. Vuong, N. Lee, Y. Liu, A. Horani, T. Huang, J. B. Case, W. Yang, M. S. Diamond, S. L. Brody, J. Dougherty, S. B. Kutluay, S. B. Kutluay, The translational landscape of SARS-CoV-2 and infected cells. bioRxiv 2020.11.03.367516 [Preprint]. 16 November 2020; https://doi.org/10.1101/2020.11.03.367516.

29. J. Rabl, M. Leibundgut, S. F. Ataide, A. Haag, N. Ban, Crystal structure of the eukaryotic 40 S ribosomal subunit in complex with initiation factor 1 . Science 331, 730-736 (2011). doi:10.1126/science.1198308 Medline

30. S. Takyar, R. P. Hickerson, H. F. Noller, mRNA helicase activity of the ribosome. Cell 120, 49-58 (2005). doi:10.1016/i.cell.2004.11.042 Medline

31. Z. Lin, R. J. C. Gilbert, I. Brierley, Spacer-length dependence of programmed -1 or -2 ribosomal frameshifting on a U6A heptamer supports a role for messenger RNA (mRNA) tension in frameshifting. Nucleic Acids Res. 40, 8674-8689 (2012). doi:10.1093/nar/gks629 Medline

32. H. Amiri, H. F. Noller, Structural evidence for product stabilization by the ribosomal mRNA helicase. RNA 25, 364-375 (2019). doi:10.1261/rna.068965.118 Medline

33. N. Caliskan, V. I. Katunin, R. Belardinelli, F. Peske, M. V. Rodnina, Programmed -1 frameshifting by kinetic partitioning during impeded translocation. Cell 157, 16191631 (2014). doi:10.1016/i.cell.2014.04.041 Medline
34. M. Bouvet, A. Lugari, C. C. Posthuma, J. C. Zevenhoven, S. Bernard, S. Betzi, I. Imbert, B. Canard, J. C. Guillemot, P. Lécine, S. Pfefferle, C. Drosten, E. J. Snijder, E. Decroly, X. Morelli, Coronavirus Nsp10, a critical co-factor for activation of multiple replicative enzymes. J. Biol. Chem. 289, 25783-25796 (2014). doi:10.1074/ibc.M114.577353 Medline

35. E. C. Smith, J. B. Case, H. Blanc, O. Isakov, N. Shomron, M. Vignuzzi, M. R. Denison, Mutations in coronavirus nonstructural protein 10 decrease virus replication fidelity. J. Virol. 89, 6418-6426 (2015). doi:10.1128/JVl.00110-15 Medline

36. S. K. Natchiar, A. G. Myasnikov, H. Kratzat, I. Hazemann, B. P. Klaholz, Visualization of chemical modifications in the human $80 \mathrm{~S}$ ribosome structure. Nature 551, 472-477 (2017). doi:10.1038/nature24482 Medline

37. J. S. Joseph, K. S. Saikatendu, V. Subramanian, B. W. Neuman, A. Brooun, M. Griffith, K. Moy, M. K. Yadav, J. Velasquez, M. J. Buchmeier, R. C. Stevens, P. Kuhn, Crystal structure of nonstructural protein 10 from the severe acute respiratory syndrome coronavirus reveals a novel fold with two zinc-binding motifs. J. Virol. 80, 7894-7901 (2006). doi:10.1128/JVI.00467-06 Medline

38. H. R. Harrington, M. H. Zimmer, L. M. Chamness, V. Nash, W. D. Penn, T. F. Miller III, S. Mukhopadhyay, J. P. Schlebach, Cotranslational folding stimulates programmed ribosomal frameshifting in the alphavirus structural polyprotein. $J$. Biol. Chem. 295, 6798-6808 (2020). doi:10.1074/ibc.RA120.012706 Medline

39. H. Nakatogawa, K. Ito, The ribosomal exit tunnel functions as a discriminating gate. Cell 108, 629-636 (2002). doi:10.1016/S0092-8674(02)00649-9 Medline

40. D. H. Goldman, C. M. Kaiser, A. Milin, M. Righini, I. Tinoco Jr., C. Bustamante, Ribosome. Mechanical force releases nascent chain-mediated ribosome arrest in vitro and in vivo. Science 348, 457-460 (2015). doi:10.1126/science.1261909 Medline

41. G. Kramer, D. Boehringer, N. Ban, B. Bukau, The ribosome as a platform for cotranslational processing, folding and targeting of newly synthesized proteins. Nat. Struct. Mol. Biol. 16. 589-597 (2009). doi:10.1038/nsmb.1614 Medline

42. K. Döring, N. Ahmed, T. Riemer, H. G. Suresh, Y. Vainshtein, M. Habich, J. Riemer, M. P. Mayer, E. P. O'Brien, G. Kramer, B. Bukau, Profiling Ssb-nascent chain interactions reveals principles of Hsp70-assisted folding. Cell 170, 298-311.e20 (2017). doi:10.1016/i.cell.2017.06.038 Medline

43. S. J. Park, Y. G. Kim, H. J. Park, Identification of RNA pseudoknot-binding ligand that inhibits the -1 ribosomal frameshifting of SARS-coronavirus by structurebased virtual screening. J. Am. Chem. Soc. 133, 10094-10100 (2011). doi:10.1021/ja1098325 Medline

44. Y. Sun, L. Abriola, Y. V. Surovtseva, B. D. Lindenbach, J. U. Guo, Restriction of SARS-CoV-2 replication by targeting programmed -1 ribosomal frameshifting in vitro. bioRxiv 2020.10.21.349225 (2020). 10.1101/2020.10.21.349225 Medline

45. A. Sharma, M. Mariappan, S. Appathurai, R. S. Hegde, In vitro dissection of protein translocation into the mammalian endoplasmic reticulum. Methods Mol. Biol. 619, 339-363 (2010). doi:10.1007/978-1-60327-412-8 20 Medline

46. S. Q. Zheng, E. Palovcak, J. P. Armache, K. A. Verba, Y. Cheng, D. A. Agard, MotionCor2: Anisotropic correction of beam-induced motion for improved cryoelectron microscopy. Nat. Methods 14, 331-332 (2017). doi:10.1038/nmeth.4193 Medline

47. K. Zhang, Gctf: Real-time CTF determination and correction. J. Struct. Biol. 193, 112 (2016). doi:10.1016/i.jsb.2015.11.003 Medline

48. J. Zivanov, T. Nakane, B. O. Forsberg, D. Kimanius, W. J. H. Hagen, E. Lindahl, S. H. W. Scheres, New tools for automated high-resolution cryo-EM structure determination in RELION-3. elife 7, e42166 (2018). doi:10.7554/eLife.42166 Medline

49. A. Punjani, J. L. Rubinstein, D. J. Fleet, M. A. Brubaker, cryoSPARC: Algorithms for rapid unsupervised cryo-EM structure determination. Nat. Methods 14, 290-296 (2017). doi:10.1038/nmeth.4169 Medline

50. A. Punjani, D. J. Fleet, 3D variability analysis: Resolving continuous flexibility and discrete heterogeneity from single particle cryo-EM. J. Struct. Biol. 213, 107702 (2021). doi:10.1016/j.jsb.2021.107702 Medline

51. T. C. Terwilliger, O. V. Sobolev, P. V. Afonine, P. D. Adams, Automated map sharpening by maximization of detail and connectivity. Acta Crystallogr. D Struct. Biol. 74, 545-559 (2018). doi:10.1107/S2059798318004655 Medline

52. S. Shao, J. Murray, A. Brown, J. Taunton, V. Ramakrishnan, R. S. Hegde, Decoding mammalian ribosome-mRNA states by translational GTPase complexes. Cell 167. 1229-1240.e15 (2016). doi:10.1016/i.cell.2016.10.046 Medline 
53. E. F. Pettersen, T. D. Goddard, C. C. Huang, G. S. Couch, D. M. Greenblatt, E. C. Meng, T. E. Ferrin, UCSF Chimera-A visualization system for exploratory research and analysis. J. Comput. Chem. 25, 1605-1612 (2004). doi:10.1002/jcc.20084 Medline

54. P. Emsley, B. Lohkamp, W. G. Scott, K. Cowtan, Features and development of Coot. Acta Crystallogr. D Biol. Crystallogr. 66, 486-501 (2010). doi:10.1107/S0907444910007493 Medline

55. D. Liebschner, P. V. Afonine, M. L. Baker, G. Bunkóczi, V. B. Chen, T. I. Croll, B. Hintze, L. W. Hung, S. Jain, A. J. McCoy, N. W. Moriarty, R. D. Oeffner, B. K. Poon, M. G. Prisant, R. J. Read, J. S. Richardson, D. C. Richardson, M. D. Sammito, O. V. Sobolev, D. H. Stockwell, T. C. Terwilliger, A. G. Urzhumtsev, L. L. Videau, C. J. Williams, P. D. Adams, Macromolecular structure determination using x-rays, neutrons and electrons: Recent developments in Phenix. Acta Crystallogr. D Struct. Biol. 75, 861-877 (2019). doi:10.1107/S2059798319011471 Medline

56. K. J. Webb, C. I. Zurita-Lopez, Q. Al-Hadid, A. Laganowsky, B. D. Young, R. S. Lipson, P. Souda, K. F. Faull, J. P. Whitelegge, S. G. Clarke, A novel 3methylhistidine modification of yeast ribosomal protein $\mathrm{Rpl} 3$ is dependent upon the YIL110W methyltransferase. J. Biol. Chem. 285, 37598-37606 (2010), doi:10.1074/jbc.M110.170787 Medline

57. A. Brown, S. Shao, J. Murray, R. S. Hegde, V. Ramakrishnan, Structural basis for stop codon recognition in eukaryotes. Nature 524, 493-496 (2015). doi:10.1038/nature14896 Medline

58. N. W. Moriarty, P. D. Adams, Iron-sulfur clusters have no right angles. Acta Crystallogr. D Struct. Biol. 75, 16-20 (2019). doi:10.1107/S205979831801519X Medline

59. P. P. Chan, T. M. Lowe, GtRNAdb 2.0: An expanded database of transfer RNA genes identified in complete and draft genomes. Nucleic Acids Res. 44, D184-D189 (2016). doi:10.1093/nar/gkv1309 Medline

60. N. K. Sinha, A. Ordureau, K. Best, J. A. Saba, B. Zinshteyn, E. Sundaramoorthy, A. Fulzele, D. M. Garshott, T. Denk, M. Thoms, J. A. Paulo, J. W. Harper, E. J. Bennett, R. Beckmann, R. Green, EDF1 coordinates cellular responses to ribosome collisions. eLife 9, e58828 (2020). doi:10.7554/eLife. 58828 Medline

61. A. W. Schüttelkopf, D. M. F. van Aalten, PRODRG: A tool for high-throughput crystallography of protein-ligand complexes. Acta Crystallogr. D Biol. Crystallogr. 60, 1355-1363 (2004). doi:10.1107/S0907444904011679 Medline

62. V. B. Chen, W. B. Arendall III, J. J. Headd, D. A. Keedy, R. M. Immormino, G. J. Kapral, L. W. Murray, J. S. Richardson, D. C. Richardson, MolProbity: All-atom structure validation for macromolecular crystallography. Acta Crystallogr. D Biol. Crystallogr. 66, 12-21 (2010). doi:10.1107/S0907444909042073 Medline

63. F. Sievers, A. Wilm, D. Dineen, T. J. Gibson, K. Karplus, W. Li, R. Lopez, H McWilliam, M. Remmert, J. Söding, J. D. Thompson, D. G. Higgins, Fast, scalable generation of high-quality protein multiple sequence alignments using Clustal Omega. Mol. Syst. Biol. 7, 539 (2011). doi:10.1038/msb.2011.75 Medline

64. X. Robert, P. Gouet, Deciphering key features in protein structures with the new ENDscript server. Nucleic Acids Res. 42, W320-W324 (2014). doi:10.1093/nar/gku316 Medline

65. G. Loughran, M. T. Howard, A. E. Firth, J. F. Atkins, Avoidance of reporter assay distortions from fused dual reporters. RNA 23, 1285-1289 (2017). doi:10.1261/rna.061051.117 Medline

66. B. W. Dyer, F. A. Ferrer, D. K. Klinedinst, R. Rodriguez, A noncommercial dual luciferase enzyme assay system for reporter gene analysis. Anal. Biochem. 282 158-161 (2000). doi:10.1006/abio.2000.4605 Medline

67. N. J. McGlincy, N. T. Ingolia, Transcriptome-wide measurement of translation by ribosome profiling. Methods 126, 112-129 (2017) doi:10.1016/i.ymeth.2017.05.028 Medline

68. M. Martin, Cutadapt removes adapter sequences from high-throughput sequencing reads. EMBnet.journal 17, 10 (2011). doi:10.14806/ej.17.1.200

69. T. Smith, A. Heger, I. Sudbery, UMI-tools: Modeling sequencing errors in Unique Molecular Identifiers to improve quantification accuracy. Genome Res. 27, 491499 (2017). doi:10.1101/gr.209601.116 Medline

70. B. Langmead, S. L. Salzberg, Fast gapped-read alignment with Bowtie 2. Nat. Methods 9, 357-359 (2012). doi:10.1038/nmeth.1923 Medline

71. R. Edgar, M. Domrachev, A. E. Lash, Gene Expression Omnibus: NCBI gene expression and hybridization array data repository. Nucleic Acids Res. 30, 207210 (2002). doi:10.1093/nar/30.1.207 Medline
72. V. G. George, J. C. Hierholzer, E. W. Ades, "Cell culture" in Virology Methods Manual, B. W. J. Mahy, H. O. Kangro, Eds. (Academic Press, 1996), pp. 3-24.

73. J. Schindelin, I. Arganda-Carreras, E. Frise, V. Kaynig, M. Longair, T. Pietzsch, S. Preibisch, C. Rueden, S. Saalfeld, B. Schmid, J. Y. Tinevez, D. J. White, V. Hartenstein, K. Eliceiri, P. Tomancak, A. Cardona, Fiji: An open-source platform for biological-image analysis. Nat. Methods 9, 676-682 (2012). doi:10.1038/nmeth.2019 Medline

74. J. Mutterer, E. Zinck, Quick-and-clean article figures with FigureJ. J. Microsc. 252 89-91 (2013). doi:10.1111/imi.12069 Medline

75. T. D. Goddard, C. C. Huang, E. C. Meng, E. F. Pettersen, G. S. Couch, J. H. Morris T. E. Ferrin, UCSF ChimeraX: Meeting modern challenges in visualization and analysis. Protein Sci. 27, 14-25 (2018). doi:10.1002/pro.3235 Medline

76. G. Cardone, J. B. Heymann, A. C. Steven, One number does not fit all: Mapping local variations in resolution in cryo-EM reconstructions. J. Struct. Biol. 184, 226236 (2013). doi:10.1016/i.jsb.2013.08.002 Medline

77. V. Chandrasekaran, S. Juszkiewicz, J. Choi, J. D. Puglisi, A. Brown, S. Shao, V. Ramakrishnan, R. S. Hegde, Mechanism of ribosome stalling during translation of a poly(A) tail. Nat. Struct. Mol. Biol. 26, 1132-1140 (2019). doi:10.1038/s41594019-0331-x Medline

78. W. A. Cantara, P. F. Crain, J. Rozenski, J. A. McCloskey, K. A. Harris, X. Zhang, F. A. P. Vendeix, D. Fabris, P. F. Agris, The RNA modification database, RNAMDB: 2011 update. Nucleic Acids Res. 39, D195-D201 (2011). doi:10.1093/nar/gkq1028 Medline

\section{ACKNOWLEDGMENTS}

We thank A. Jomaa for advice on cryo-EM data processing, Noreen Casey for support with technical resources and A. Picenoni for help with the grid preparation. We are indebted to the ETH scientific center for optical and electron microscopy (ScopeM) for access to electron microscopes, and in particular to M. Peterek and D. Boehringer. We thank Junjie Guo for helpful discussions and for providing merafloxacin compound. Funding: This work was supported by the Swiss National Science Foundation (SNSF) to NB and VT (grant 31003A_182341 and 310030_173085) via the National Center of Excellence in RNA and Disease (project funding 182880) to N. Ban, V. Thiel and D. Gatfield; The Federal Ministry of Education and Research, Germany (BMBF; grant RAPID, \#01KI1723A) to AK and VT; The Irish Research Council Advanced Laureate (IRCLA/2019/74) to J.F.A., who thanks Prof. G.F. Fitzgerald for funds from Carbery Group Ltd. Author contributions: PRB and NB initiated the project, and together with JFA designed the experiments. PRB carried out biochemical experiments and sample preparation. AS and PRB prepared grids. AS carried out data collection and calculated EM maps. AS and ML performed molecular model building and refinement. AS, ML, PRB and NB interpreted the structure. PRB, AS, ML and NB drafted the manuscript. AS prepared the figures. GL and KMOC performed dualluciferase reporter assays. AM prepared and purified the drug-like ligand MTDB. AK and VT formulated experiments to test viral infected cells. AK carried out testing of ligand on viral-infected cells. RM, RD and DG designed, carried out, analyzed and interpreted ribosome profiling experiments. All authors contributed to the final version of the manuscript. Competing interests: The authors declare no competing interest. Data and materials availability: The structure and cryo-EM map for the high-resolution reconstruction is available in the Protein Data Bank (PDB) as PDB ID 707Y and in the Electron Microscopy Data Bank (EMDB) as EMD-12756, respectively. The structure refined into the further classified particle set reconstruction and the corresponding maps are available as PDB-707Z and EMD-12757, respectively. The structure of the ribosome bound with eRF1 (AAQ) and ABCE1 reconstruction and the corresponding maps are available as PDB-7080 and EMD-12758, respectively. The structure of the colliding ribosome reconstruction and the corresponding maps are available as PDB-7081 and EMD-12759, respectively. Structure of the composite map of the disome is available in the EMDB as EMD-12760. Ribosome profiling data have been deposited at GSE167421. This work is licensed under a Creative Commons Attribution 4.0 International (CC BY 4.0) license, which permits unrestricted use, distribution, and reproduction in any medium, provided the original work is properly cited. To view a copy of this license, visit https:/creativecommons.org/licenses/by/4.0/. This license does not apply to figures/photos/artwork or other content included in the article that is credited 
to a third party; obtain authorization from the rights holder before using such material.

\section{SUPPLEMENTARY MATERIALS}

science.sciencemag.org/cgi/content/full/science.abf3546/DC1

Materials and Methods

Figs. S1 to S11

Tables S1 to S4

References (44-78)

MDAR Reproducibility Checklist

20 October 2020; resubmitted 24 February 2021

Accepted 7 May 2021

Published online 13 May 2021

10.1126/science.abf3546 

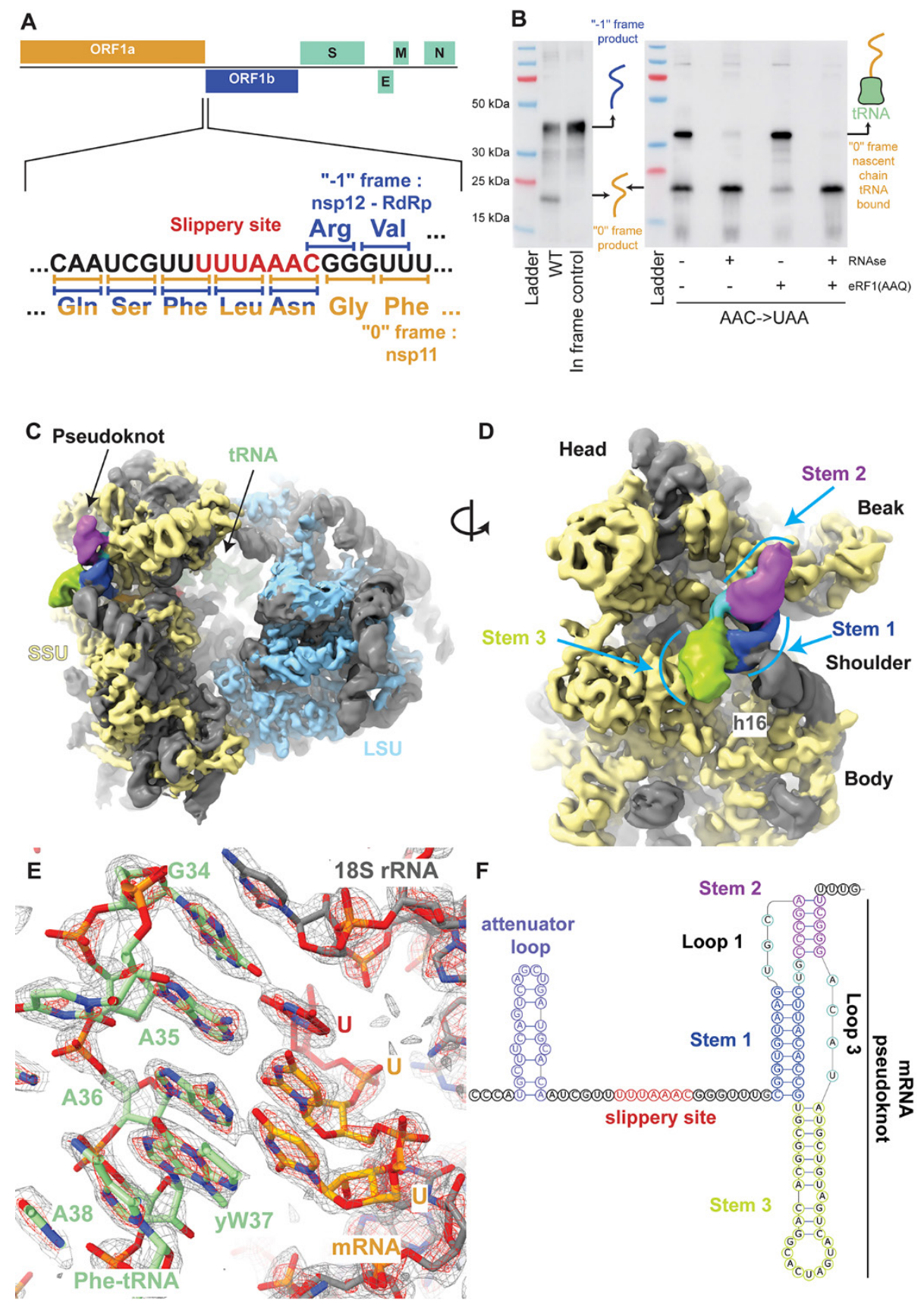
Fig. 1. The SARS-CoV-2 pseudoknot interacts with the ribosome and pauses translation upstream of the slippery site. (A) Schematic of the SARS-CoV-2 main ORF. In the close up view of the frameshift event, codons and corresponding amino acids are shown. During -1 frameshifting, the 'slippery site' codons UUA (Leu) and AAC (Asn) are the last codons decoded in the 0 frame. Upon -1 frameshifting of the AAC codon to AAA, translation resumes at the CGG (Arg) triplet, where elongation proceeds uninterrupted to produce full-length Nsp12. (B) In vitro translation reaction depicting pausing at the frameshift site. Efficient frameshifting is observed for the WT template, consistent with our dual luciferase assays (see methods). Samples for cryo-EM originally intended to be trapped by dominant negative eRF1 (AAQ) show a tRNA-bound pause in proximity of the frameshift site. The tRNA-associated band is lost upon RNase treatment. Reactions without added eRF1 (AAQ) produce a similarly paused product. (C) Overview of the density low pass filtered to $6 \AA$ with the pseudoknot found close to the entry of the mRNA channel on the small subunit (SSU). The SSU proteins are colored in yellow, the large subunit (LSU) proteins in blue and the rRNA in grey. The pseudoknot is colored according to its secondary structure as in (F), and the P-site tRNA is colored in green. (D) Close-up view of the pseudoknot from the solvent-exposed side of the SSU. Helix h16 of the 18S rRNA interacts with the base of Stem 1. Unpaired loop-forming nucleotides are colored in cyan. (E) P-site codon-anticodon interactions reveal a Phe (UUU) codon interacting with tRNA(Phe). (F) Schematic of the revised secondary structure elements in the pseudoknot necessary for -1 PRF with different functional regions labeled and colored accordingly. 


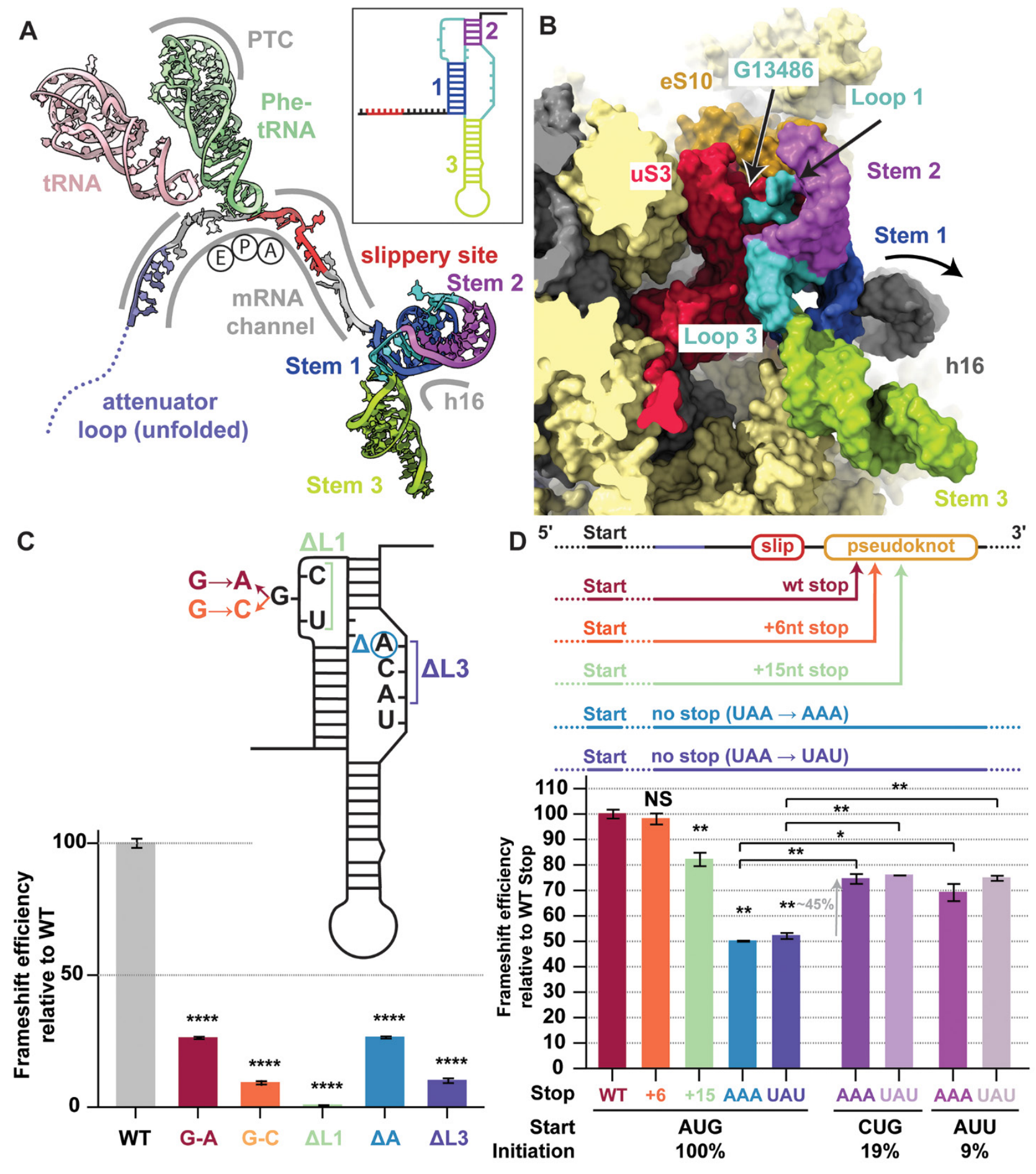


Fig. 2. Critical features of the ribosome-bound pseudoknot. (A) Overview of the frameshift-primed state. The stimulatory pseudoknot pauses the ribosome at the penultimate codon (UUU) of the slippery site (red), with P- (green) and E- (pink) sites occupied by tRNAs, and an empty A-site awaiting decoding in the non-rotated state. The length of the spacer region (grey) is critical for exact positioning of the pseudoknot as the spacer exerts tension at the entry of the mRNA channel (fig. S6C). (B) The backbone of Loop 1 (UGC) (cyan) of the pseudoknot interacts with the N-terminal domain of uS3 (red) and the C-terminal tail of eS10 (orange). mRNA residue G13486 is flipped out and interacts with uS3 (fig. S6D). (C) Mutagenesis experiments using dual luciferase assays in HEK293T cells indicate that the G13486 interaction is specific. Mutation of G13486 to other residues leads to a marked reduction in frameshifting efficiency, and deletion of Loop 1 ( $\triangle$ L1) completely abolishes frameshifting. Similarly, deletion of a single nucleotide (A13537) in Loop 2 reduces frameshifting, while deletion of the entire loop $(\Delta \mathrm{L} 2)$ abolishes frameshifting. Normalized (Firefly/Renilla) luciferase activities were calculated for each construct as a percentage of their individual normalized in-frame controls. Data are presented as mean values \pm standard deviations of three biological replicates (sets of translation reactions) averaged after three measurements, with error bars representing standard deviations. (D) Mutagenesis experiments using dual luciferase reporter assays in HEK293T cells show that the position of the 0 frame stop codon influences frameshifting. Leaving the pseudoknot unaltered, incremental increase in the distance of the 0 frame stop codon from the frameshift site leads to a concomitant decrease in frameshifting levels. Loss of the stop codon in 0 frame leads to a sharp decline in frameshifting levels. This reduction is rescued by $\sim 45 \%$ upon decreasing ribosome loading levels by implementing weaker initiation codons. The graph is normalized relative to the WT frameshifting of $25 \%$. Mutations and complementary mutations are shown in fig. S8. 


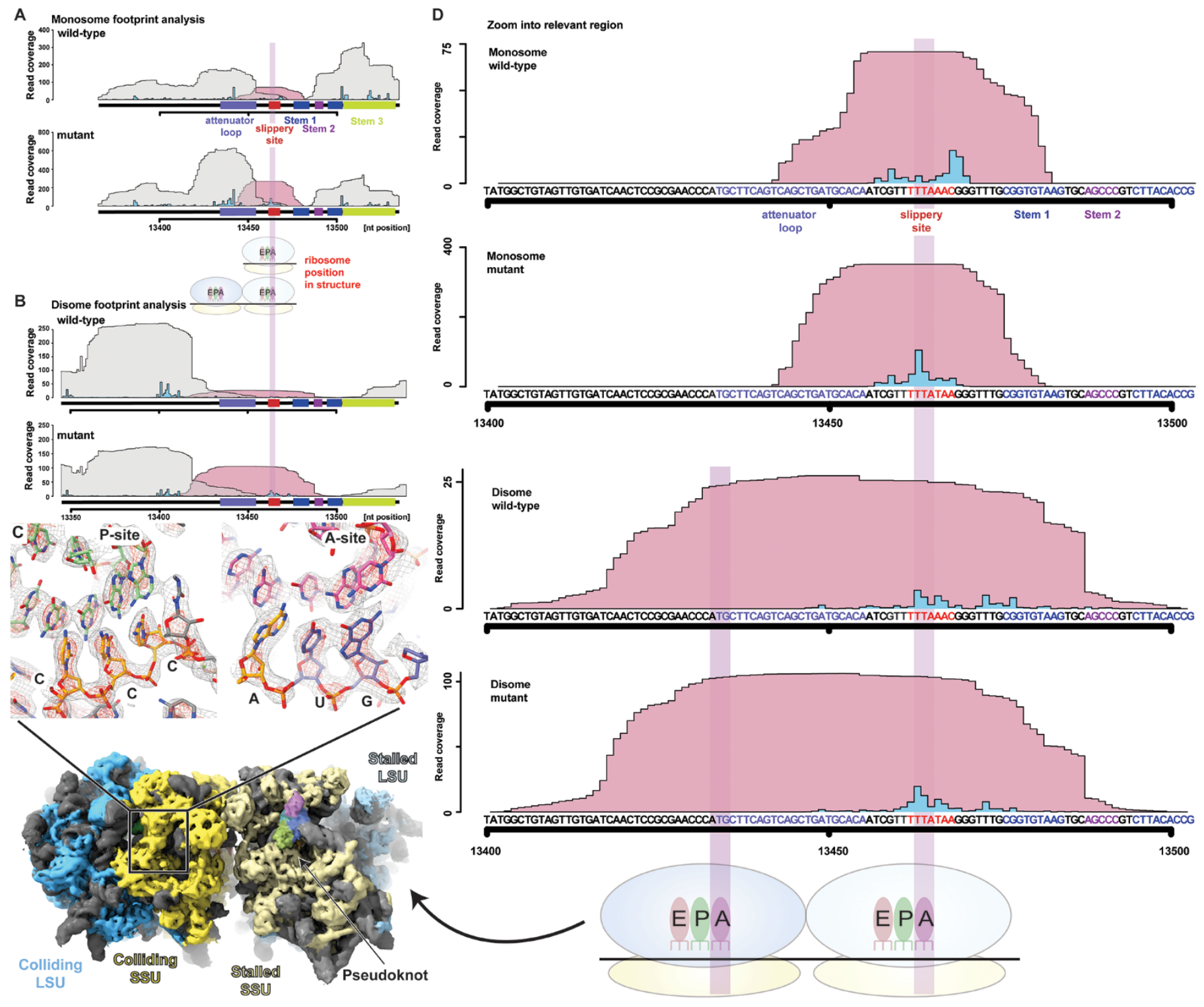

Fig. 3. Pseudoknot-mediated pause occurs prior to the -1 frameshifting event. (A and $B$ ) Footprint coverage for WT and mutant constructs determined by monosome- and disome-selective ribosome profiling. Pileup of reads from the indicated areas are plotted separately for reads that overlap (pink) vs. do not overlap (grey) the frameshift site (indicated by red bar below $\mathrm{x}$-axis). The predicted A-sites of the ribosomes giving rise to the footprints are depicted as blue peaks. A-site predictions were carried out as described in Supplementary Material. (C) Zoom into the frameshift region from (A) and $(B)$ reveals that monosome profiles show transient occupancy in the vicinity of the frameshift site, while disome profiles - indicative of strong pause sites - show a similarly enhanced occupancy at the first codon (UUA) of the frameshift site in both WT and mutant constructs. A-site codons of the leading and trailing ribosome are highlighted with a translucent bar and correspond to those seen in the disome structure in (D). (D) In high resolution cryo-EM reconstructions of disomes at the frameshift site, the P-and A-sites of the trailing ribosome show occupancy of CCC and AUG codons, respectively, corresponding to the positions estimated by disome profiling. Disome maps were calculated by separately refining the orientational parameters for each ribosome. 

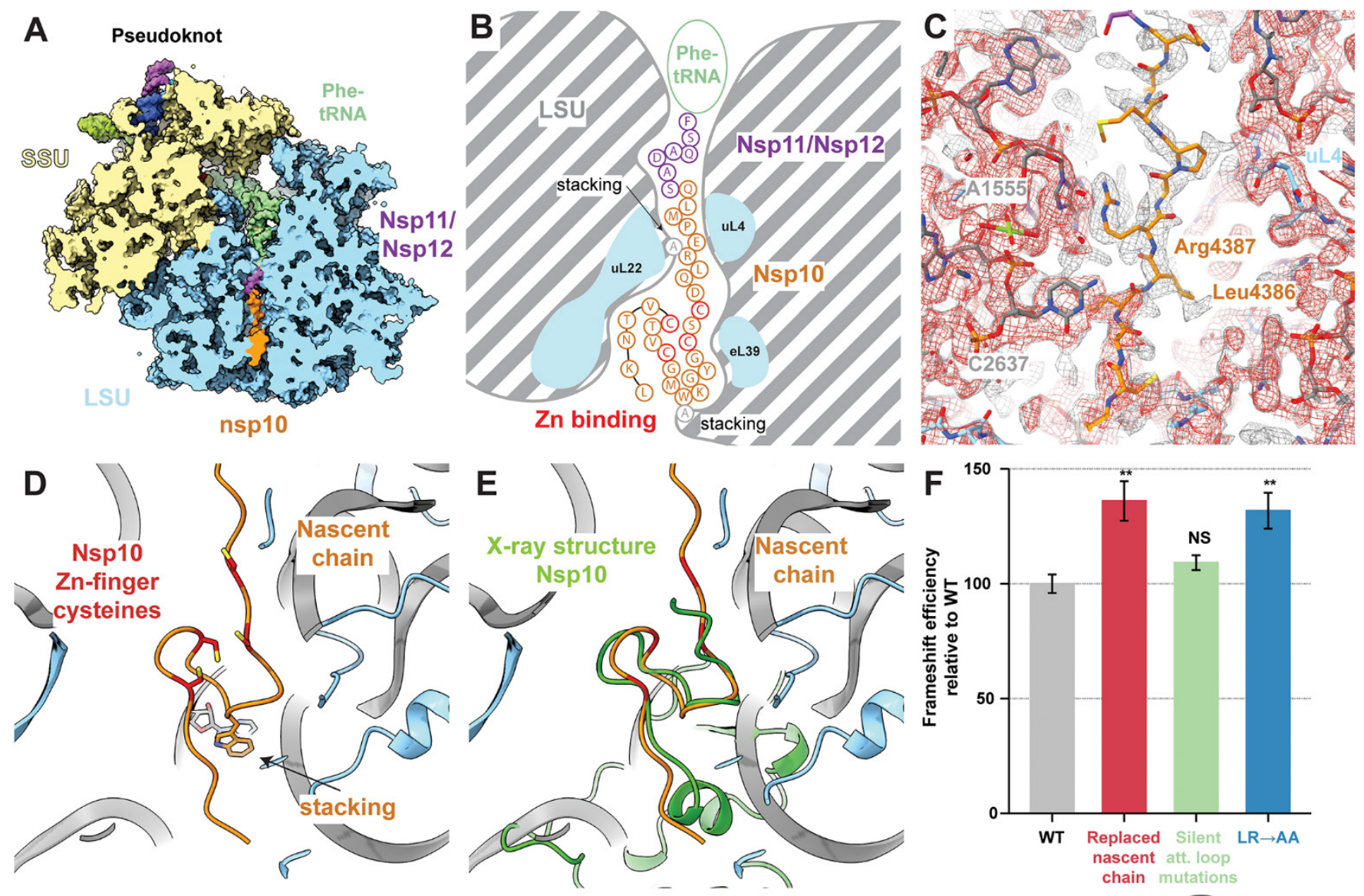

G

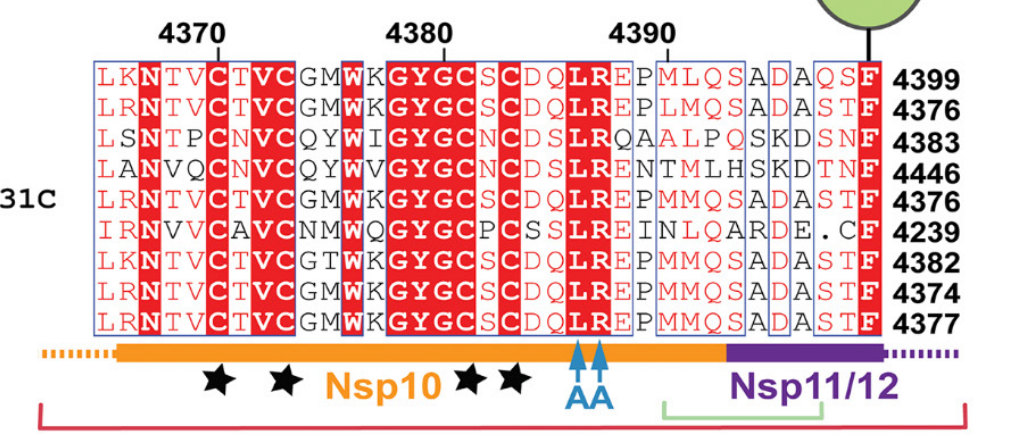

4380

SARS2_coronavirus

SARS_coronavirus

MERS_coronavirus

Hedgehog_coronavirus_1

Bat_SARS-like_coronavirus_YNLF_31C

Bat_coronavirus_HKU9

Bat_coronavirus_279/2005

Bat_coronavirus_Rp3/2004

Bat_coronavirus_Cp/Yunnan2011

Exchange to non-viral nascent chain

Silent mutations in

attenuator loop 
Fig. 4. The nascent viral polypeptide co-translationally folds and specifically interacts with the ribosomal tunnel. (A) Cross-section of the pseudoknot-paused ribosome structure showing the exit tunnel. The nascent C terminus of Nsp10 (orange) and the $N$ terminus of Nsp11/12 (purple) are visible from the PTC to the periphery of the ribosome exit tunnel (LSU in blue). (B) Schematic representation of the path of nascent peptide along the exit tunnel. Arg 4387 stacks with 28S rRNA residue $\mathrm{A} 1555$ at the constriction site. Further down, where the tunnel widens, the C-terminal zinc finger domain of Nsp10 folds co-translationally, with Trp 4376 stacking on A2261 of 28S rRNA. (C) Well-ordered density is visible for Arg 4387 of Nsp10 as it stacks onto A1555 of 28S rRNA at the constriction site and is stabilized by Leu 4386. The structure is shown within the cryo-EM map contoured at two different levels (grey and red). ( $D$ and $E$ ) The overlay of the co-translationally folded zinc finger domain with the crystal structure of Nsp10 [green, PDB 2FYG (37)] reveals the structural similarity. (F) Probing the role of nascent chain interactions with the ribosome exit tunnel using an $\mathrm{RRL}$ in vitro system. Mutations of the interacting residues were tested for their effect on frameshifting shown in comparison to the wild type frameshifting (41\% frameshifting was normalized to $100 \%$ ). Replacement of the entire nascent chain with an unrelated sequence leads to a 35\% relative increase in frameshifting, which is only in part due to loss of the $5^{\prime}$ attenuator loop. Interactions around the constriction site likely serve to attenuate frameshifting, as replacement of the interacting Arg 4387 and stabilizing Leu 4386 with Ala increases frameshifting by 30\%. (G) Alignment of SARS2 with closely related sequences of other coronaviruses highlighting the conservation of the mutated residues [colored as in (F)]. The shown sequence stretch encompasses the C-terminal zinc finger domain of Nsp10 (orange) and parts of Nsp11/Nsp12 (purple) visible in our reconstruction. Nascent chain residues Leu 4386 and Arg 4387 interacting with the ribosomal exit tunnel are strictly conserved, while the conservation of neighboring residues is lower. Stars represent the four cysteines of the Nsp10 zinc finger. 


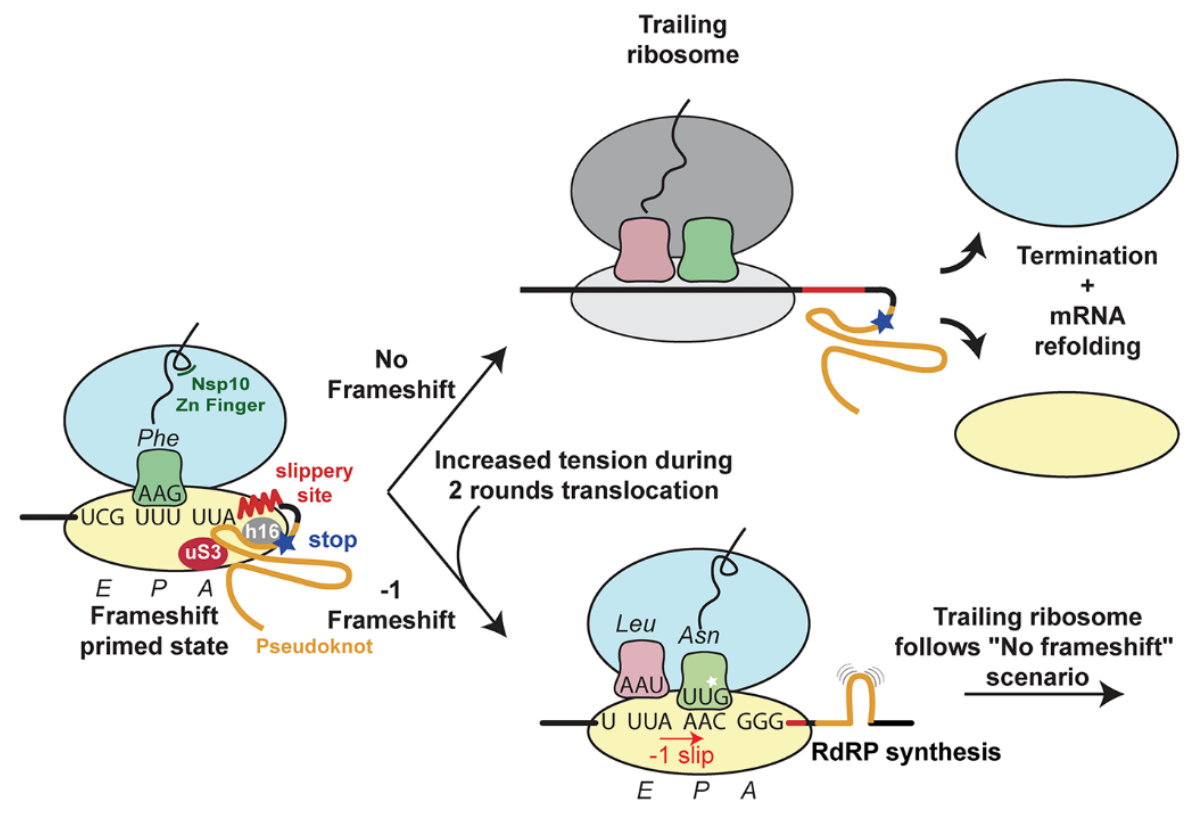

Fig. 5. Structure based model for -1 programmed frameshifting in coronaviruses and its regulation. The observed interactions between the pseudoknot and the ribosome prime the system for frameshifting. The features of the pseudoknot and the interactions between the nascent chain and the ribosomal tunnel play a role in the efficiency of frameshifting. The efficiency of frameshifting is increased by the presence of a stop codon near the frameshifting site. Ribosomes that progress beyond the frameshifting site in the 0 frame quickly terminate and disassemble, thereby increasing the chances that the pseudoknot will refold before it is encountered by the closely trailing ribosome. The trailing ribosome in turn encounters the pseudoknot, which increases the possibility of undergoing -1 frameshifting. 